\title{
FOXM1 regulates glycolysis in hepatocellular carcinoma by transactivating glucose transporter 1 expression
}

\author{
RUNZE SHANG ${ }^{*}$, MENG PU $^{1 *}, \mathrm{YU} \mathrm{LI}^{2}$ and DESHENG WANG ${ }^{1}$ \\ ${ }^{1}$ Department of Hepatobiliary Surgery, Xijing Hospital, Fourth Military Medical University, Xi'an, Shaanxi 710032; \\ ${ }^{2}$ Cell Engineering Research Center and Department of Cell Biology, State Key Laboratory of Cancer Biology, \\ Fourth Military Medical University, Xi'an, Shaanxi 710032, P.R. China
}

Received August 3, 2016; Accepted October 31, 2016

DOI: $10.3892 /$ or.2017.5472

\begin{abstract}
The Forkhead box M1 (FOXM1) transcription factor plays crucial roles in the initiation and progression of various malignancies, including hepatocellular carcinoma (HCC). However, the mechanism by which FOXM1 regulates cancer metabolism remains unclear. In the present study, overexpression and RNA interference (RNAi) approaches were used to investigate the role of FOXM1 in the regulation of glycolysis in vitro. Luciferase reporter assays were used to explore the transcriptional regulation of the glucose transporter 1 (GLUT1) promoter by FOXM1. Then, immunohistochemical staining was used to examine the expression of FOXM1 and GLUT1 in 100 paired HCC and adjacent non-cancerous liver tissues. Chi-square test and logistic regression analysis were performed to evaluate the association between FOXM1 and GLUT1 expression with clinicopathological characteristics. Our data showed that FOXM1 promoted glycolysis in the HCC cells. FOXM1 knockdown significantly reduced the expression of GLUT1 among key glycolysis-related molecules in the different HCC cell lines. Glucose uptake and lactate production assay showed that FOXM1 positively regulated glycolysis based on GLUT1 expression. Moreover, FOXM1 overexpression increased and knockdown decreased GLUT1 expression. Luciferase reporter assays showed that the -206 to $-199 \mathrm{bp}$ region of the GLUT1
\end{abstract}

Correspondence to: Professor Desheng Wang, Department of Hepatobiliary Surgery, Xijing Hospital, Fourth Military Medical University, 127 West Changle Street, Xi'an, Shaanxi 710032, P.R. China

E-mail: hepato@fmmu.edu.cn

Professor Yu Li, Cell Engineering Research Center and Department of Cell Biology, State Key Laboratory of Cancer Biology, Fourth Military Medical University, 169 West Changle Street, Xi'an, Shaanxi 710032, P.R. China

E-mail: liyufmmu@fmmu.edu.cn

*Contributed equally

Key words: FOXM1, GLUT1, hepatocellular carcinoma, glycolysis promoter is important for FOXM1 to enhance GLUT1 promoter activity. The results of the IHC analysis showed that the protein expression of FOXM1 and GLUT1 was closely related to the tumor histological grade and TNM stage. In addition, GLUT1 expression was also related to microvascular invasion. In conclusion, overexpression of FOXM1 and GLUT1 may play critical roles in HCC. FOXM1 promotes HCC glycolysis by transactivating GLUT1 expression.

\section{Introduction}

Hepatocellular carcinoma (HCC) is one of the most prevalent neoplasms and the second leading cause of cancer-related mortality worldwide (1). Despite the application of surgical resection, ablation, embolization and other therapeutic methods, the overall prognosis of patients with $\mathrm{HCC}$ remains poor and the incidence of HCC is increasing annually $(2,3)$. Therefore, the underlying mechanisms that promote the pathogenesis of this deadly disease must be further investigated.

Metabolic reprogramming has long been linked to cancer. One of the classical theories of metabolic abnormality in tumors is the Warburg effect, which describes increased glycolysis even under normal oxygen conditions (4). The shift of the metabolic pathway from oxidative phosphorylation to glycolysis is more rapid to meet the demands necessary for the process of tumor progression. However, it is inefficient to generate ATP by consuming units of glucose under glycolysis conditions (5). Therefore, transformed cells need to uptake more glucose than normal cells. The glucose transporter 1 (GLUT1) isoform is a key rate-limiting protein in the transport of glucose and is overexpressed in HCC (6). However, the mechanism underlying increased glycolysis by targeting GLUT1 in HCC is unknown.

The Forkhead box M1 (FOXM1) transcriptional factor is a member of the Forkhead family that is involved in cell proliferation and cell cycle progression. The expression of FOXM1 is frequently upregulated in many solid tumors (7). The overexpression of FOXM1 promotes proliferation, invasion and epithelial-mesenchymal transition, and is associated with a poor prognosis in HCC (8-10). Recent studies also revealed that FOXM1 promotes glucose consumption, lactate production and increased lactate dehydrogenase $\mathrm{A}$ (LDHA) activity in different cells, suggesting a pivotal role 
for FOXM1 in the regulation of cell aerobic glycolysis $(11,12)$. However, a mechanistic understanding of the involvement of FOXM1 in HCC metabolism is lacking.

In the present study, we demonstrated that high expression of FOXM1 promotes the reprogramming of glucose metabolism in human HCC cells through the transactivation of GLUT1 expression, which results in the malignant progression of liver cancer.

\section{Materials and methods}

Cell culture. All human HCC cell lines (HepG2, Hep3B, MHCC-97H and SMMC-7721) used in the present study were obtained from the Shanghai Cellular Biology Center (Shanghai, China). HCC cells were cultured in Dulbecco's modified Eagle's medium (DMEM) culture-medium supplemented with $10 \%$ fetal bovine serum (FBS; HyClone, Logan, UT, USA), penicillin $(100 \mathrm{U} / \mathrm{ml})$ and streptomycin $(100 \mu \mathrm{g} / \mathrm{ml})$ at $37^{\circ} \mathrm{C}$ in an atmosphere of humidified air containing $5 \% \mathrm{CO}_{2}$.

Patient samples. In the present study, 100 samples were obtained at the time of surgery at the Department of Hepatobiliary Surgery, Xijing Hospital between 2005 and 2013. Clinicopathological information (age, gender, tumor size, histological grade, liver cirrhosis, hepatitis B virus infection, microvascular invasion and TNM stage) for each patient was collected from medical records. Two pathologists confirmed the pathologic diagnoses of all slides. The protocol of the present study was approved by the Ethics Committee of Xijing Hospital. The informed written consent to participate in the present study was obtained from each patient.

FOXM1 and GLUT1 detection by immunohistochemistry (IHC).Paraffin-embedded HCC samples and their surrounding liver tissues were used to construct the tissue microarrays (in collaboration with Xi'an Dong-Ao Biosciences Co., Ltd. (Xi'an, China). The TMA was dyed to detect FOXM1 and GLUT1 expression by IHC. In brief, the slides were deparaffinized and rehydrated in xylene and alcohol solutions and heated in boiling sodium citrate buffer $(10 \mathrm{mM}, \mathrm{pH}$ 6.0). After being maintained at a sub-boiling temperature for $20 \mathrm{~min}$, the slides were incubated in $3 \%$ hydrogen peroxide for $15 \mathrm{~min}$ for endogenous peroxidase inactivation. Then, the sections were blocked with $5 \%$ normal goat serum at room temperature for $1 \mathrm{~h}$. Subsequently, the slides were incubated with rabbit anti-FOXM1 (ProteinTech Group, Wuhan, China) or with GLUT1 (Thermo Fisher Scientific, Inc., Waltham, MA, USA) polyclonal antibody overnight at $4^{\circ} \mathrm{C}$. After a phosphatebuffered saline (PBS) rinse, the sections were incubated with horseradish peroxidase (HRP)-conjugated goat anti-rabbit antibody (Zhongshan Goldenbridge Biotechnology Co., Ltd., Beijing, China) according to the manufacturer's instructions. Then, the slides were incubated with fresh 0.05\% 3,3'-diaminobenzidine (DAB) for several minutes and counterstained with hematoxylin. Two pathologists independently examined the IHC staining. The scores were estimated according to both the staining intensity and the number of stained cells, as previously described (13). Briefly, the staining intensity was scored as: 0 , negative; 1 , weak; and 2 , strong. The staining extent was determined according to the percentage of positive
Table I. Primers for the RT-PCR.

Gene $\quad$ Primers $\left(5^{\prime}-3^{\prime}\right)$

\begin{tabular}{|c|c|}
\hline FOXM1 & $\begin{array}{l}\text { F 5'-GGGCGCACGGCGGAAGATGAA3' } \\
\text { R 5'-CCACTCTTCCAAGGGAGGGCTC3' }\end{array}$ \\
\hline ALDO & $\begin{array}{l}\text { F 5'-ATGCCACTCTCAACCTCAATGCTATC-3' } \\
\text { R 5'-TTATTTTCTTGGGTGGGTATTCTGG-3' }\end{array}$ \\
\hline ENDOLASE & $\begin{array}{l}\text { F 5'-CTGATGCTGGAGTTGGATGG-3' } \\
\text { R 5'-CCATTGATCACGTTGAAGGC-3' }\end{array}$ \\
\hline GLUT1 & $\begin{array}{l}\text { F 5'-GGCCAAGAGTGTGCTAAAGAA-3' } \\
\text { R 5'-ACAGCGTTGATGCCAGACAG-3' }\end{array}$ \\
\hline GLUT4 & $\begin{array}{l}\text { F 5'-CTTCATCATTGGCATGGGTTT-3' } \\
\text { R 5'-CGGGTTTCAGGCACTTTTAGG-3' }\end{array}$ \\
\hline GAPDH & $\begin{array}{l}\text { F 5'-CAGCCTCAAGATCATCAGCA-3' } \\
\text { R 5'-TGTGGTCATGAGTCCTTCCA-3' }\end{array}$ \\
\hline G6PI & $\begin{array}{l}\text { F 5'-AGGCTG CTGCCACATAAGGT-3' } \\
\text { R 5'-AGCGTCGTGAGAGGTCACTTG-3' }\end{array}$ \\
\hline HK2 & $\begin{array}{l}\text { F 5'-GATTTCACCAAGCGTGGACT-3' } \\
\text { R 5'-CCACACCCACTGTCACTTTG-3' }\end{array}$ \\
\hline LDHA & $\begin{array}{l}\text { F 5'-CAGCTTGGAGTTTGCAGTTAC-3' } \\
\text { R 5'-TGATGGATCTCCAACATGG-3' }\end{array}$ \\
\hline LDHB & $\begin{array}{l}\text { F 5'-CCTAGAGCTCACTAGTCACAG-3' } \\
\text { R 5'-CTCCTGTGCAAAATGGCAAC-3' }\end{array}$ \\
\hline PFK-L & $\begin{array}{l}\text { F 5'-GGACAGGAAAGAGGAAGTGAC-3' } \\
\text { R 5'-CGTAGATGAGGAAGACTTTGGC-3' }\end{array}$ \\
\hline PFK-M & $\begin{array}{l}\text { F 5'-ATTCGGGCTGTGTTCTGG-3' } \\
\text { R 5'-TGGCTAGGATTTTGAGGATGG-3' }\end{array}$ \\
\hline PFK-P & $\begin{array}{l}\text { F 5'-CATCGACAATGATTTCTGCGG-3' } \\
\text { R 5'-CCATCACCTCCAGAACGAAG-3' }\end{array}$ \\
\hline PGAM1 & $\begin{array}{l}\text { F 5'-CCTGGAGAACCGCTTC-3' } \\
\text { R 5'-CATGGGCTGCAATCAGTACAC-3' }\end{array}$ \\
\hline PGK1 & $\begin{array}{l}\text { F 5'-CGGTAGTCCTTATGAGCC-3' } \\
\text { R 5'-CATGAAAGCGGAGGTTCT-3' }\end{array}$ \\
\hline PKM1 & $\begin{array}{l}\text { F 5'-CTATCCTCTGGAGGCTGTGC-3' } \\
\text { R 5'-CCATGAGGTCTGTGGAGTGA-3' }\end{array}$ \\
\hline PKM2 & $\begin{array}{l}\text { F 5'-GGGTTCGGAGGTTTGATG-3' } \\
\text { R 5'-ACGGCGGTGGCTTCTGT-3' }\end{array}$ \\
\hline$\beta$-actin & $\begin{array}{l}\text { F 5'-TAGTTGCGTTACACCCTTTCTTG-3' } \\
\text { R 5'-TCACCTTCACCGTTCCAGTTT-3' }\end{array}$ \\
\hline
\end{tabular}

F, forward; R, reverse. FOXM1, Forkhead box M1; LDHA, lactate dehydrogenase A; GLUT1, glucose transporter 1.

staining cells: 0 , negative; $1,1-25 \% ; 2,26-50 \% ; 3,51-75 \%$; and $4,76-100 \%$. Therefore, positive expression was defined as a total score of $>3$, while negative expression was defined as a total score of $\leq 3$.

RNA extraction, cDNA synthesis and quantitative real-time $P C R$. TRIzol reagent (Invitrogen, Carlsbad, CA, USA) was 
A

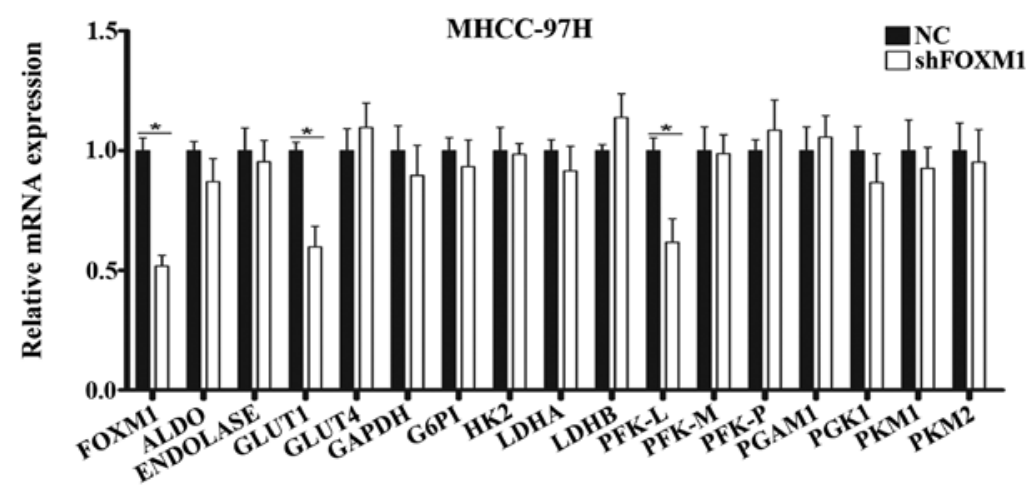

B

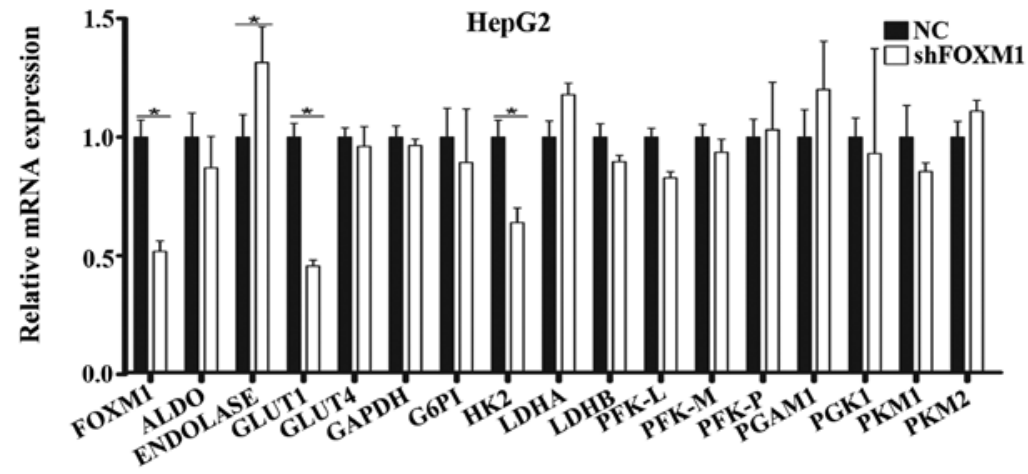

C

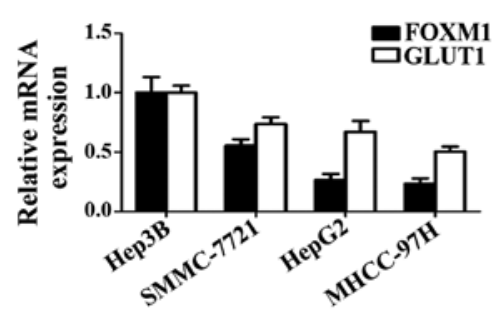

$\mathbf{E}$

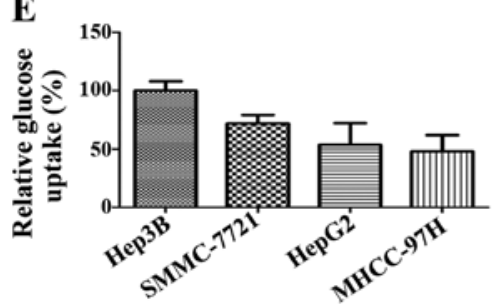

D

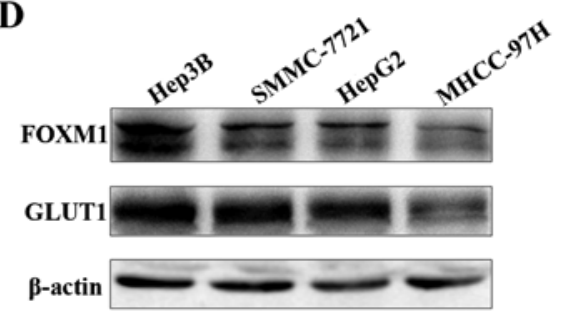

F

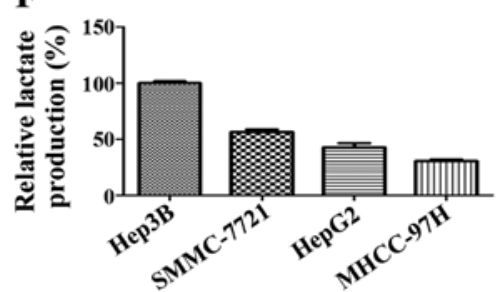

Figure 1. FOXM1 regulates HCC glycolysis and glycolytic molecules. (A and B) RT-qPCR analysis of the mRNA expression of glycolytic transporters and enzymes in HepG2 and MHCC-97H cells after knockdown of FOXM1 expression. The expression of GLUT1 was significantly upregulated in both cell lines. (C and D) Relative FOXM1 and GLUT1 expression in human HCC cell lines Hep3B, SMMC-7721, HepG2 and MHCC-97H, shown by (C) RT-qPCR and (D) western blotting. (E) The relative glucose uptake and (F) lactate production level were consistent with the relative FOXM1 and GLUT1 expression in the different HCC cell lines; ${ }^{P}<<0.05$. HCC, hepatocellular carcinoma.

used to extracted the total RNA of the HCC cells according to the procedure recommended by the manufacturer. Reverse transcription was conducted with PrimeScript ${ }^{\mathrm{TM}}$ Master Mix (Takara Bio, Dalian, China). Then, quantitative real-time PCR was performed with SYBR Premix Ex Taq ${ }^{\mathrm{TM}}$ II (Takara) and detected on the Bio-Rad IQ5 (Bio-Rad Laboratories, Hercules, CA, USA) system. $\beta$-actin was used as the reference, and the $\Delta \Delta \mathrm{Ct}$ method was used to analyze the gene expression using iQ5 Optical System Software (Bio-Rad Laboratories). The sequences of RT-qPCR primers used for mRNA analysis in the present study are listed in Table I.
shRNAs and plasmid transfection. The shRNA of FOXM1 and the shRNA-NC not targeting any known mammalian gene were synthesized by Shanghai GenePharma Co. (Shanghai, China). The plasmid pcDNA3.1-FOXM1 and control vector pcDNA3.1 were previously described (14). Transfection of shRNAs and plasmids was performed with Lipofectamine 2000 (Invitrogen) according to the manufacturer's instructions.

Western blotting. Ice-cold PBS was used to wash the cells 3 times. Then, protein was extracted using strong RIPA buffer (Beyotime, Shanghai, China) with protease inhibitor 
(Thermo Fisher Scientific, Waltham, MA, USA). The lysates were separated by $8 \%$ SDS-PAGE and then transferred to nitrocellulose membranes. After blocking with $5 \%$ milk for $1 \mathrm{~h}$, the membranes were incubated with primary antibodies diluted in $5 \%$ milk overnight at $4^{\circ} \mathrm{C}$. HRP-conjugated secondary antibody (Abcam, Cambridge, MA, USA) was incubated with the membranes for $1 \mathrm{~h}$ at room temperature. After washing with Tris-buffered saline with Tween-20 (TBST), the blots were visualized with the ChemiDoc ${ }^{\mathrm{TM}} \mathrm{XRS}+$ using Image $\mathrm{Lab}^{\mathrm{TM}}$ software (Bio-Rad Laboratories).

Glucose uptake and lactate production assays. The glucose assay kit (BioVision, Milpitas, CA, USA) was used to detect relative glucose uptake of cells before and after transfection in accordance with the manufacturer's instructions. The relative lactate production among different groups was measured via using the lactate assay kit (BioVision) according to the protocol.

Luciferase reporter assay. Cells were incubated at $37^{\circ} \mathrm{C}$ overnight in 6-well plates. Each group was co-transfected with GLUT1 promoter reporters, shFOXM1 or pcDNA3.1-FOXM1 plasmid. Twenty-four hours later, whole-cell lysates were prepared with passive lysis buffer. Then, the collections were used for reporter detection using the Dual-Luciferase Reporter System (Promega, Madison, WI, USA) and the reactions were measured.

Statistical analysis. All statistical analyses were performed using SPSS 17.0 statistical software (SPSS, Inc., Chicago, IL, USA). Student's t-test was performed for two group comparisons. One-way ANOVA and Dunnett's multiple comparison test were used for multiple group comparisons. The expression of FOXM1 and GLUT1 in HCC samples and their relationship with clinicopathological characteristics were analyzed using $\chi^{2}$ tests. A $\mathrm{P}<0.05$ was considered to indicate a statistically significant result.

\section{Results}

FOXM1 promotes glycolysis in HCC cells. As previously reported, numerous oncogenes and tumor-suppressor genes participate in cancer metabolism by regulating the expression of key metabolism-related molecules. To investigate the role of FOXM1 in HCC glycolysis, we evaluated the effect of FOXM1 on the expression of glycolysis-related molecules. Among these key glycolysis-related molecules, we found that only GLUT1 mRNA expression was significantly downregulated in both the HepG2 and MHCC-97H HCC cell lines when FOXM1 was knocked down by shRNA (Fig. 1A and B). To further confirm the effect of FOXM1 on GLUT1 expression and HCC glycolysis, we assessed the correlation of FOXM1 expression with GLUT1 expression, glucose uptake and lactate production in 4 different HCC cell lines. As shown in Fig. 1C-F, expression of FOXM1 was tightly associated with the expression of GLUT1, glucose uptake and lactate production.

FOXM1 positively regulates the Warburg effect. The Warburg effect is characterized by consuming more glucose and
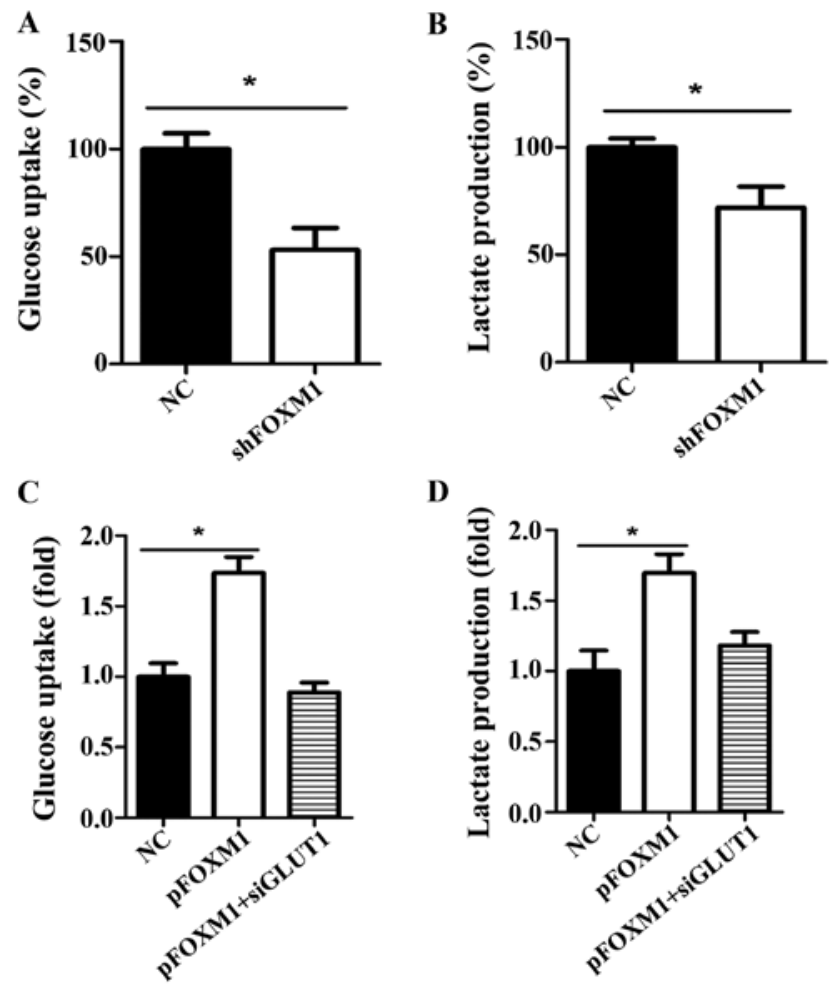

D

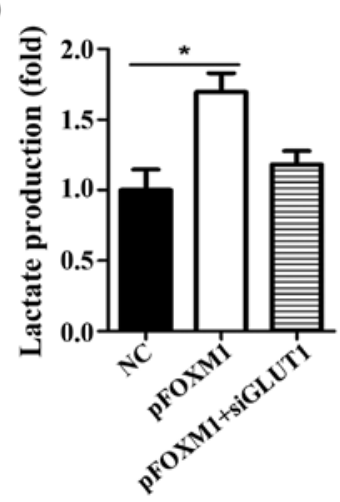

Figure 2. FOXM1 regulates glucose uptake and lactate production in HCC cells. (A and B) Hep3B cells were transfected with the shRNA-NC or shFOXM1 plasmid. (C and D) MHCC-97H cells were transfected with pcDNA3.1 control, pcDNA3.1-FOXM1, or pcDNA3.1-FOXM1 and siGLUT1. (A and C) The glucose uptake and (B and D) lactate production in the HCC cells in the different groups were determined after a 24-h incubation; ${ }^{*} \mathrm{P}<0.05$. HCC, hepatocellular carcinoma.

producing more lactate than normal cells when the glycolysis pathway is activated in cancer cells. To investigate whether FOXM1 impacts the Warburg effect in HCC, we detected the level of glucose uptake and lactate production when the expression of FOXM1 was altered using shRNA or an overexpression plasmid. We knocked down FOXM1 expression in Hep3B cells, which have a relatively high expression of FOXM1 among the 4 HCC cell lines used in the present study. We found that the decreased expression of FOXM1 significantly reduced glucose uptake and lactate production in the Hep3B cells (Fig. 2A and B). In contrast, overexpression of FOXM1 in MHCC-97H cells, which have the lowest FOXM1 expression of the HCC cells, significantly increased glucose uptake and lactate production (Fig. 2C and D). Moreover, the increased glucose uptake and lactate production caused by FOXM1 expression was decreased when GLUT1 was knocked down using a specific siRNA. These results suggest that FOXM1 positively regulates the Warburg effect in HCC cells, which is dependent on the expression of GLUT1.

FOXM1 promotes GLUT1 expression. To further determine whether FOXM1 regulates the expression of GLUT1, RT-qPCR and western blot assay were used. Upon downregulation of FOXM1, GLUT1 expression was decreased at both the mRNA and protein levels in the Hep3B cells compared with the blank and negative control groups (Fig. 3A, C and E). By comparison, upregulation of the FOXM1 in MHCC-97H cells promoted the expression of GLUT1 (Fig. 3B, D and F). 

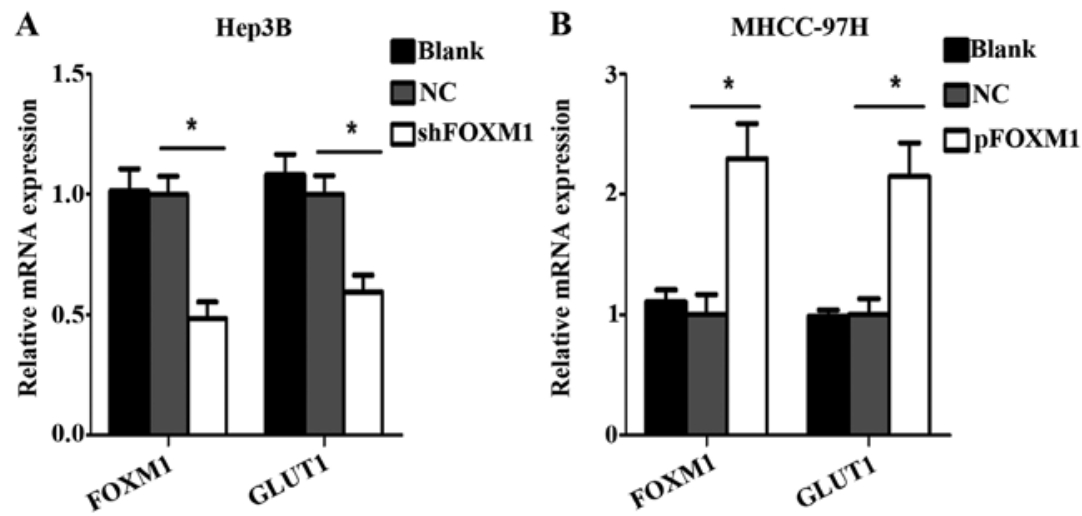

C

D
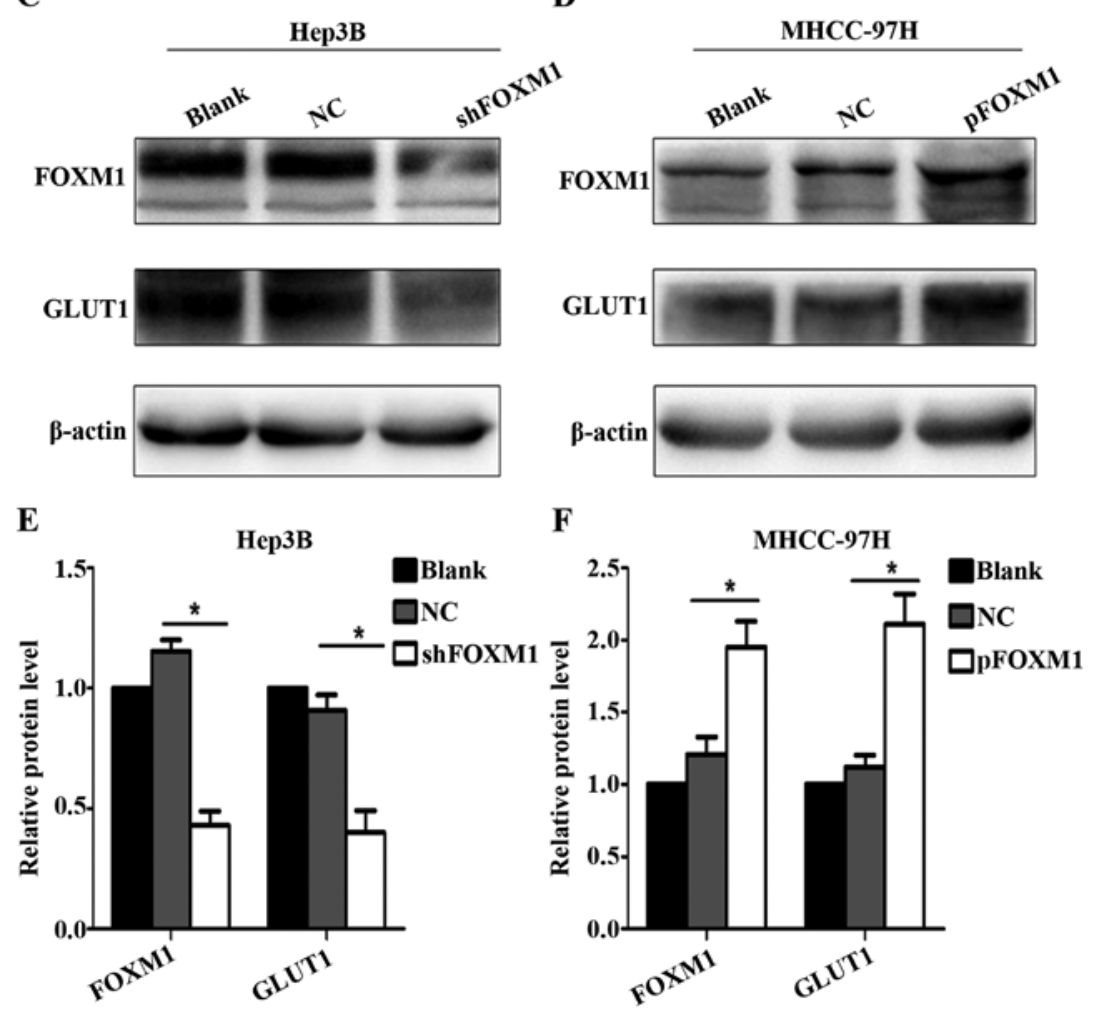

Figure 3. FOXM1 regulates GLUT1 expression. (A and B) Quantitation of GLUT1 mRNA levels in Hep3B and MHCC97-H cells by qRT-PCR after FOXM1 knockdown or overexpression. (C and D) Detection of GLUT1 protein in Hep3B and MHCC97-H cells by western blot analysis after FOXM1 knockdown or overexpression. (E and F) The bar charts show the ratio of FOXM1 and GLUT1 protein to $\beta$-actin by densitometry in the different groups; " $\mathrm{P}<0.05$.

GLUT1 is a direct target of FOXM1. To further verify whether GLUT1 is a direct target gene of FOXM1 transcription activation, we analyzed the sequences of the GLUT1 promoter and identified a putative FOXM1-binding site in its promoter region (Fig. 4A) (12). Then, 3 reporter gene constructs were produced based on the potential binding site. These reporter constructs were co-transfected with pcDNA3.1-FOXM1, shFOXM1 or control vector into HCC cells, and the promoter activity was then measured. As shown in Fig. 4B and D, the altered expression of FOXM1 significantly changed the activity of the GLUT1 promoter in the P558 construct, but not in the P102 construct, which did not contain the putative FOXM1 binding site. As shown in Fig. 4C and E, FOXM1 knockdown or overexpression significantly reduced or increased respectively the activity of the wild-type pLuc-GLUT1 construct in the Hep3B and MHCC-97H cells. However, the altered expression of FOXM1 did not change the activity of the mutant pLuc-GLUT1 construct.

Expression of FOXM1 and GLUT1 in HCC and adjacent liver tissues. To explore the expression of FOXM1 and GLUT1 in HCC, 100 HCC patients were enrolled in the present study. We found that FOXM1 was mainly expressed in the cytoplasm as well as the nucleus, whereas GLUT1 was exclusively expressed in plasma membranes in the HCC patients (Fig. 5). Positive staining for FOXM1 in the HCC and adjacent normal tissues was $64 / 100$ and $35 / 100$, respectively, and $24 / 100$ and $0 / 100$ for GLUT1, respectively.

We further showed that the FOXM1 and GLUT1 genes were also increased at the transcriptional level. The real-time quantitative RT-PCR assay demonstrated that the mRNA expression level of FOXM1 and GLUT1 were significantly 


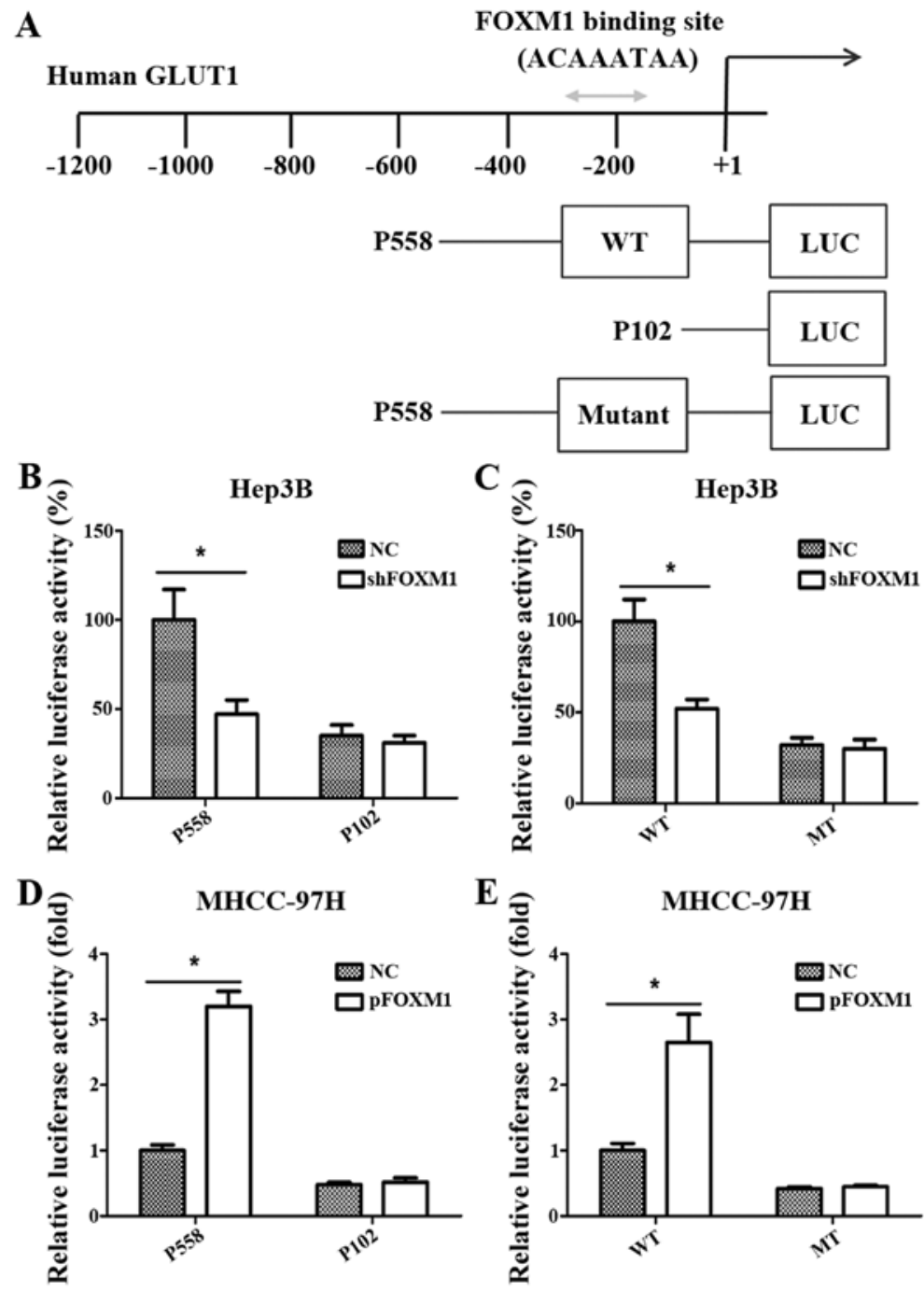

Figure 4. GLUT1 is a direct target of FOXM1 in HCC cells. (A) Predicted FOXM1 binding site in the promoter region of FOXM1. A wild-type pLuc-GLUT1 construct P558, a mutant pLuc-GLUT1 construct and a P102 construct (without the putative FOXM1 binding site) were generated. (B) A shFOXM1 or negative control and a P558 or P102 vector were introduced into Hep3B cells, and the relative luciferase activity was measured. (C) Co-transfection of shFOXM1 or negative control and a wild-type or mutant P558 vector was carried out in the Hep3B cells, and the relative luciferase activity was measured. (D) A pCDNA3.1-FOXM1 or pCDNA3.1-control and a P558 or P102 vector were introduced into MHCC-97H cells, and the relative luciferase activity was measured (E) Co-transfection of pCDNA3.1-FOXM1 or pCDNA3.1-control and a wild-type or mutant P558 vector in the Hep3B cells, and the relative luciferase activity was measured; " $\mathrm{P}<0.05$.

Table II. Correlation analysis of FOXM1 and GLUT1 expression in HCC cases.

\begin{tabular}{|c|c|c|c|c|c|}
\hline & \multirow{2}{*}{$\begin{array}{l}\text { No. of } \\
\text { patients }\end{array}$} & \multicolumn{2}{|c|}{ GLUT1 expression } & \multirow[b]{2}{*}{$\mathrm{r}$} & \multirow[b]{2}{*}{ P-value } \\
\hline & & Negative & Positive & & \\
\hline \multicolumn{6}{|l|}{$\begin{array}{l}\text { FOXM1 } \\
\text { expression }\end{array}$} \\
\hline Negative & 36 & 36 & 0 & 0.421 & $<0.001^{\mathrm{a}}$ \\
\hline Positive & 64 & 40 & 24 & & \\
\hline
\end{tabular}

Statistical analyses were performed using Pearson's $\chi^{2}$ test. ${ }^{\mathrm{a}}<0.05$ is considered significant. FOXM1, Forkhead box M1; GLUT1, glucose transporter 1 .

increased in the HCC tissues compared to the levels noted in the adjacent non-cancerous tissues (Fig. 6A and B).
Correlation between FOXM1 and GLUT1 in HCC. We evaluated the correlation between FOXM1 and GLUT1 expression at both the mRNA and protein levels. Our data showed a positive correlation between the expression of FOXM1 and GLUT1 ( $\mathrm{r}=0.483, \mathrm{P}=0.0016$ ) at the mRNA level (Fig. 6C). We also analyzed the results of the HCC staining and also found a positive correlation between FOXM1 and GLUT1 expression at the protein level (Table II). Notably, further statistical analysis demonstrated that the nuclear localization of FOXM1 was more closely associated with GLUT1 expression, which suggests a crucial role of FOXM1 in GLUT1 transactivation (Table III).

Correlation between FOXM1 and GLUT1 expression with the clinicopathological features. As shown in Table IV, the expression of FOXM1 and GLUT1 was significantly associated with tumor histological grade and TNM stage in HCC. In addition, GLUT1 expression was also related to microvascular invasion. 

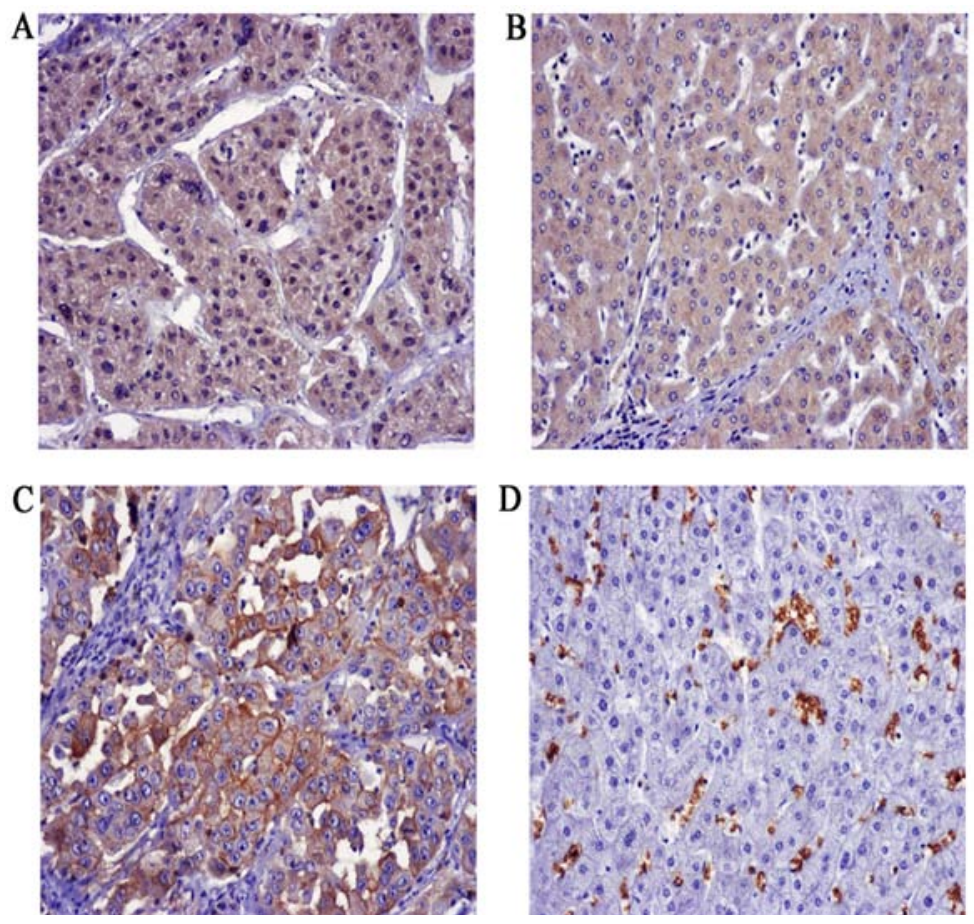

Figure 5. Expression analysis of FOXM1 and GLUT1 in HCC tissues using immunohistochemistry. (A) Positive staining for FOXM1 in HCC tissue. (B) Positive staining for FOXM1 in adjacent non-tumorous tissue. (C) Positive staining for GLUT1 in HCC tissue. (D) Negative staining for GLUT1 in adjacent non-tumorous tissue. SP, magnification, x200.

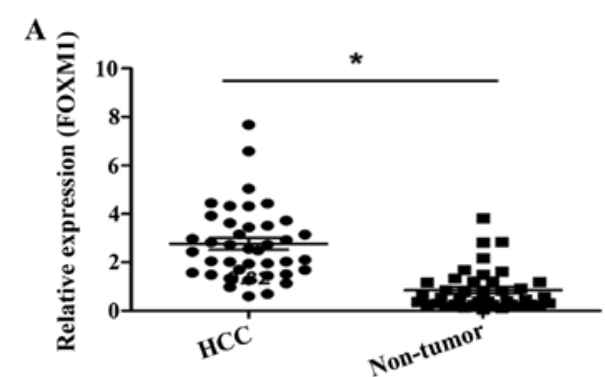

B

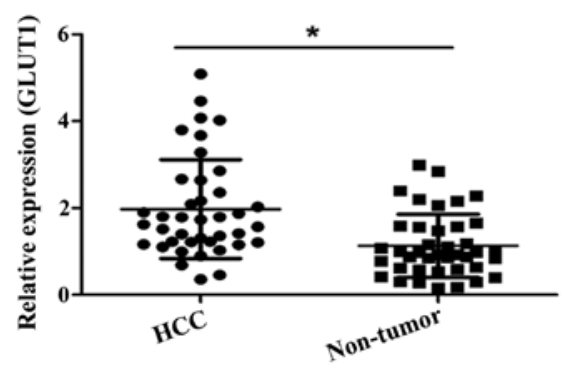

C

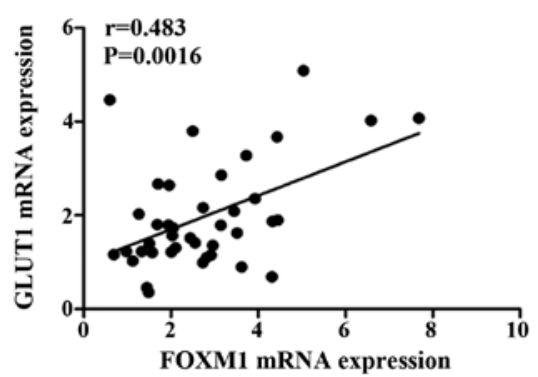

Figure 6. FOXM1 expression is positively correlated with GLUT1 expression at the mRNA level in HCC tissues. (A and B) FOXM1 and GLUT1 mRNA were detected in $40 \mathrm{HCC}$ tissues and in matched adjacent non-cancerous liver tissues by qRT-PCR. (C) Spearman's correlation analysis between FOXM1 and GLUT1 mRNA levels in HCC tissues $(\mathrm{r}=0.483, \mathrm{P}=0.0016) ;{ }^{*} \mathrm{P}<0.05$. $\mathrm{HCC}$, hepatocellular carcinoma.
Table III. Correlation analysis of GLUT1 and FOXM1 nuclear expression in FOXM1-positive staining samples.

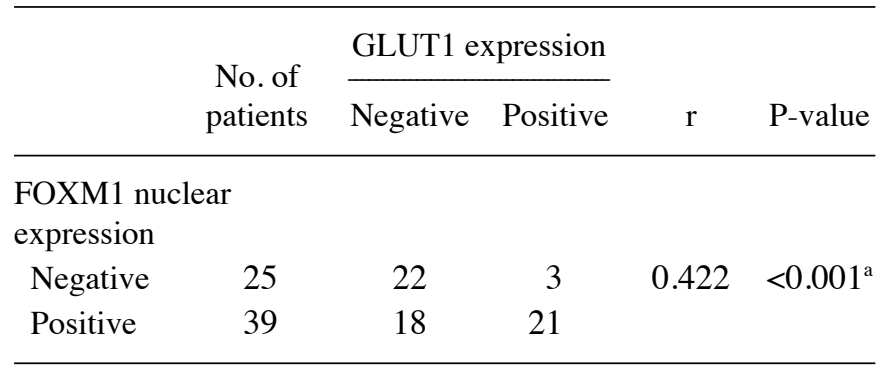

Statistical analyses were performed using Pearson's $\chi^{2}$ test. ${ }^{a} \mathrm{P}<0.05$ is considered significant. FOXM1, Forkhead box M1; GLUT1, glucose transporter 1 .

\section{Discussion}

FOXM1 is a proliferation related transcription factor, which plays crucial roles in normal cell proliferation, organogenesis and cancer progression (15). The aberrant expression of FOXM1 is involved in cancer proliferation $(16,17)$, epithelialmesenchymal transition, metastasis (10) and stem cell features (18) in hepatocellular carcinoma (HCC). However, the significance of FOXM1 in HCC glycolysis has not been established. In the present study, we assessed the role of FOXM1 in HCC glucose metabolism. The glucose uptake and lactate production assays demonstrated that FOXM1 expression was consistent with glycolysis levels in several HCC cell lines. Furthermore, we found that knockdown or overexpression of FOXM1 significantly suppressed or promoted glycolysis in HCC cells, respectively. 
Table IV. Correlation between FOXM1 and GLUT1 expression levels and clinicopathological features of the HCC patients.

\begin{tabular}{|c|c|c|c|c|c|c|c|c|c|}
\hline \multirow[b]{2}{*}{ Characteristics } & \multirow{2}{*}{$\begin{array}{c}\text { No. of } \\
\text { patients }\end{array}$} & \multicolumn{2}{|c|}{ FOXM1 expression } & \multirow[b]{2}{*}{$\mathrm{r}$} & \multirow[b]{2}{*}{ P-value } & \multirow[b]{2}{*}{$\mathrm{r}$} & \multicolumn{2}{|c|}{ GLUT1 expression } & \multirow[b]{2}{*}{ P-value } \\
\hline & & Negative & Positive & & & & Negative & Positive & \\
\hline \multicolumn{10}{|l|}{ Gender } \\
\hline Male & 74 & 29 & 45 & -0.112 & 0.344 & 60 & 14 & -0.201 & 0.062 \\
\hline Female & 26 & 7 & 19 & & & 16 & 10 & & \\
\hline \multicolumn{10}{|l|}{ Age (years) } \\
\hline$<45$ & 27 & 9 & 18 & 0.034 & 0.817 & 20 & 7 & 0.027 & 0.796 \\
\hline$\geq 45$ & 73 & 27 & 46 & & & 56 & 17 & & \\
\hline \multicolumn{10}{|c|}{ Histological grade } \\
\hline G1 & 15 & 11 & 4 & 0.275 & $0.004^{\mathrm{a}}$ & 15 & 0 & 0.326 & $0.005^{\mathrm{a}}$ \\
\hline G2 & 69 & 21 & 48 & & & 53 & 16 & & \\
\hline G3 & 16 & 4 & 12 & & & 8 & 8 & & \\
\hline \multicolumn{10}{|l|}{ Tumor size (cm) } \\
\hline$<5$ & 35 & 17 & 18 & 0.192 & 0.080 & 28 & 7 & 0.069 & 0.625 \\
\hline$\geq 5$ & 65 & 19 & 46 & & & 48 & 17 & & \\
\hline \multicolumn{10}{|l|}{ Liver cirrhosis } \\
\hline No & 39 & 15 & 24 & 0.041 & 0.831 & 32 & 7 & 0.113 & 0.339 \\
\hline Yes & 61 & 21 & 40 & & & 44 & 17 & & \\
\hline \multicolumn{10}{|l|}{ HBsAg status } \\
\hline Negative & 75 & 27 & 48 & $\leq 0.001$ & 1.000 & 56 & 19 & -0.054 & 0.788 \\
\hline Positive & 25 & 9 & 16 & & & 20 & 5 & & \\
\hline \multicolumn{10}{|c|}{ Microvascular invasion } \\
\hline No & 72 & 30 & 42 & 0.189 & 0.067 & 60 & 12 & 0.275 & $0.009^{\mathrm{a}}$ \\
\hline Yes & 28 & 6 & 22 & & & 16 & 12 & & \\
\hline \multicolumn{10}{|l|}{ TNM stage } \\
\hline I-II & 31 & 20 & 11 & 0.398 & $<0.001^{\mathrm{a}}$ & 29 & 2 & 0.275 & $0.005^{\mathrm{a}}$ \\
\hline III-IV & 69 & 16 & 53 & & & 47 & 22 & & \\
\hline
\end{tabular}

Statistical analyses were performed using Pearson's $\chi^{2}$ test. ${ }^{\text {a }}<0.05$ is considered significant. HBsAg hepatitis B surface antigen, TNM tumor-node-metastasis; FOXM1, Forkhead box M1; GLUT1, glucose transporter 1 HCC, hepatocellular carcinoma.

To determine whether FOXM1 promotes the Warburg effect in HCC, we further explored the underlying molecular mechanism of FOXM1 regulation of HCC glycolysis. A previous study showed that oncogenes and tumor-suppressor genes participate in cancer metabolism via the dysregulation of key metabolism-related molecules, including glycolysis-related transporters (GLUTs and MCTs) and glycolytic enzymes (G6PI and HK2). For example, HIF, either alone or together with the oncogene MYC, was shown to promote glycolysis by activating glucose transporters, glycolytic enzymes and lactate dehydrogenase A (LDHA) (5,19-21). Therefore, we detected the expression changes of all glycolysis-related molecules in HepG2 and MHCC-97H cell lines when FOXM1 was knocked down. We found that knockdown of FOXM1 expression did not change the expression of most of the molecules, except for GLUT1, in the different HCC cell lines. Further experiments confirmed that FOXM1 promotes HCC glycolysis by regulation of the expression of GLUT1. As previously published, FOXM1 is a typical transcription factor that belongs to the Forkhead box family, which is evolutionarily conserved and is defined by having a common DNA-binding domain called Forkhead or winged-helix domain (22). We further assessed whether FOXM1 affects GLUT1 expression via transcriptional regulation. A series of luciferase assays verified the direct transactivating role of FOXM1 on GLUT1. Taken together, our data strongly support the hypothesis that FOXM1 promotes glycolysis in HCC by transactivating GLUT1 expression.

Tumor metabolism is an important link between the tumor microenvironment and tumor progression (23). As described previously, cancer cells use the glycolysis pathway to generate ATP as well as the byproduct lactate. The accumulation of lactate in the extracellular matrix (ECM) causes the acidification of the tumor microenvironment. The acidic environment is favorable for the activation of MMPs, uPA and other critical proteases to facilitate degradation of the ECM and, subsequently, tumor invasion and metastasis. Additionally, the aberrant expression of glycolytic transporters and enzymes has 
been linked to tumor progression (24). Therefore, we evaluated the association between FOXM1 and GLUT1 expression with several clinicopathological characteristics to determine whether FOXM1 and GLUT1 expression contributes to HCC progression. The statistical analysis showed a significant association between the expression of FOXM1 and GLUT1 with the tumor histological grade and TNM stage in HCC. In addition, GLUT1 expression was also related to microvascular invasion. These results indicate that the overexpression of FOXM1 and GLUT1 may play important roles in HCC progression.

Although, our experiments investigated the regulatory role and mechanism of FOXM1 on glycolysis in HCC cells, the present study has some limitations. Firstly, the number of patients enrolled in the present study was relatively limited. Therefore, large scale studies are needed to validate these findings in the future. In addition, further experiments are required to validate the role of the FOXM1-GLUT1 axis in HCC glycolysis in vivo.

In summary, the present study showed that FOXM1 promotes glycolysis in HCC. We demonstrated that FOXM1 binds to the GLUT1 promoter to regulate the expression of GLUT1 in HCC cells. Moreover, overexpression of FOXM1 and GLUT1 plays an important role in HCC progression. Collectively, these results suggest that the FOXM1-GLUT1 axis is a potential therapeutic target in $\mathrm{HCC}$.

\section{Acknowledgements}

We thank Fuqin Zhang for advice on performing the experiments. The present study was supported by grants from the National Nature Science Foundation of China (nos. 81270549 and 30872480).

\section{References}

1. David F and Jacques F: The global and regional burden of cancer. In: World Cancer Report 2014. Bernard WS and Christopher PD (eds). International Agency for Research on Cancer, Paris, pp16-53, 2014.

2. Llovet JM: Updated treatment approach to hepatocellular carcinoma. J Gastroenterol 40: 225-235, 2005.

3. Villanueva A: Rethinking future development of molecular therapies in hepatocellular carcinoma: A bottom-up approach. J Hepatol 59: 392-395, 2013.

4. Warburg O: On the origin of cancer cells. Science 123: 309-314, 1956.

5. Cairns RA, Harris IS and Mak TW: Regulation of cancer cell metabolism. Nat Rev Cancer 11: 85-95, 2011.

6. Amann T, Maegdefrau U, Hartmann A, Agaimy A, Marienhagen J, Weiss TS, Stoeltzing O, Warnecke C, Schölmerich J, Oefner PJ, et al: GLUT1 expression is increased in hepatocellular carcinoma and promotes tumorigenesis. Am J Pathol 174: 1544-1552, 2009.
7. Zona S, Bella L, Burton MJ, Nestal de Moraes G and Lam EW: FOXM1: An emerging master regulator of DNA damage response and genotoxic agent resistance. Biochim Biophys Acta 1839: 1316-1322, 2014

8. Wu QF, Liu C, Tai MH, Liu D, Lei L, Wang RT, Tian M and Lü Y: Knockdown of FoxM1 by siRNA interference decreases cell proliferation, induces cell cycle arrest and inhibits cell invasion in MHCC-97H cells in vitro. Acta Pharmacol Sin 31: 361-366, 2010.

9. Park HJ, Gusarova G, Wang Z, Carr JR, Li J, Kim KH, Qiu J, Park YD, Williamson PR, Hay N, et al: Deregulation of FoxM1b leads to tumour metastasis. EMBO Mol Med 3: 21-34, 2011.

10. Meng FD, Wei JC, Qu K, Wang ZX, Wu QF, Tai MH, Liu HC, Zhang RY and Liu C: FoxM1 overexpression promotes epithelial-mesenchymal transition and metastasis of hepatocellular carcinoma. World J Gastroenterol 21: 196-213, 2015.

11. Davis DB, Lavine JA, Suhonen JI, Krautkramer KA, Rabaglia ME, Sperger JM, Fernandez LA, Yandell BS, Keller MP, Wang IM, et al: FoxM1 is up-regulated by obesity and stimulates beta-cell proliferation. Mol Endocrinol 24: 1822-1834, 2010.

12. Cui J, Shi M, Xie D, Wei D, Jia Z, Zheng S, Gao Y, Huang S and Xie K: FOXM1 promotes the warburg effect and pancreatic cancer progression via transactivation of LDHA expression. Clin Cancer Res 20: 2595-2606, 2014.

13. Xia L, Mo P, Huang W, Zhang L, Wang Y, Zhu H, Tian D, Liu J, Chen Z, Zhang Y, et al: The TNF- $\alpha /$ ROS/HIF-1-induced upregulation of FoxMI expression promotes HCC proliferation and resistance to apoptosis. Carcinogenesis 33: 2250-2259, 2012.

14. Liu M, Dai B, Kang SH, Ban K, Huang FJ, Lang FF, Aldape KD, Xie TX, Pelloski CE, Xie K, et al: FoxM1B is overexpressed in human glioblastomas and critically regulates the tumorigenicity of glioma cells. Cancer Res 66: 3593-3602, 2006.

15. Laoukili J, Stahl M and Medema RH: FoxM1: At the crossroads of ageing and cancer. Biochim Biophys Acta 1775: 92-102, 2007.

16. Yu M, Tang Z, Meng F, Tai M, Zhang J, Wang R, Liu C and Wu Q: Elevated expression of FoxM1 promotes the tumor cell proliferation in hepatocellular carcinoma. Tumour Biol 37: 1289-1297, 2016

17. Hu C, Liu D, Zhang Y, Lou G, Huang G, Chen B, Shen X, Gao M, Gong W, Zhou P, et al: LXR $\alpha$-mediated downregulation of FOXM1 suppresses the proliferation of hepatocellular carcinoma cells. Oncogene 33: 2888-2897, 2014.

18. Kopanja D, Pandey A, Kiefer M, Wang Z, Chandan N, Carr JR, Franks R, Yu DY, Guzman G, Maker A, et al: Essential roles of FoxM1 in Ras-induced liver cancer progression and in cancer cells with stem cell features. J Hepatol 63: 429-436, 2015.

19. Goda $\mathrm{N}$ and Kanai M: Hypoxia-inducible factors and their roles in energy metabolism. Int J Hematol 95: 457-463, 2012.

20. Kim JW, Tchernyshyov I, Semenza GL and Dang CV: HIF-1-mediated expression of pyruvate dehydrogenase kinase: A metabolic switch required for cellular adaptation to hypoxia. Cell Metab 3: 177-185, 2006.

21. Semenza GL, Roth PH, Fang HM and Wang GL: Transcriptional regulation of genes encoding glycolytic enzymes by hypoxiainducible factor 1. J Biol Chem 269: 23757-23763, 1994.

22. Wierstra I and Alves J: FOXM1, a typical proliferation-associated transcription factor. Biol Chem 388: 1257-1274, 2007.

23. Kroemer G and Pouyssegur J: Tumor cell metabolism: Cancer's Achilles' heel. Cancer Cell 13: 472-482, 2008.

24. Han T, Kang D, Ji D, Wang X, Zhan W, Fu M, Xin HB and Wang JB: How does cancer cell metabolism affect tumor migration and invasion? Cell Adh Migr 7: 395-403, 2013. 